\title{
A bright $z=5.2$ lensed submillimeter galaxy in the field of Abell 773
}

\section{HLSJ091828.6+514223}

\author{
F. Combes ${ }^{1}$, M. Rex ${ }^{2}$, T. D. Rawle ${ }^{2}$, E. Egami ${ }^{2}$, F. Boone ${ }^{3}$, I. Smail ${ }^{4}$, J. Richard ${ }^{5}$, R. J. Ivison ${ }^{6}$, M. Gurwell ${ }^{7}$, \\ C. M. Casey $^{8}$, A. Omont ${ }^{9}$, A. Berciano Alba ${ }^{10}$, M. Dessauges-Zavadsky ${ }^{11}$, A. C. Edge ${ }^{4}$, G. G. Fazio ${ }^{7}$, J.-P. Kneib ${ }^{12}$, \\ N. Okabe ${ }^{13}$, R. Pelló ${ }^{3}$, P. G. Pérez-González ${ }^{14}$, D. Schaerer ${ }^{11,3}$, G. P. Smith ${ }^{15}$, A. M. Swinbank ${ }^{4}$, and P. van der Werf ${ }^{16}$ \\ 1 Observatoire de Paris, LERMA, CNRS, 61 Av. de l'Observatoire, 75014 Paris, France \\ e-mail: francoise.combes@obspm.fr \\ 2 Steward Observatory, University of Arizona, 933 North Cherry Avenue, Tucson, AZ, 85721, USA \\ 3 Université de Toulouse, UPS-OMP, CNRS, IRAP, 9 Av. colonel Roche, BP 44346, 31028 Toulouse Cedex 4, France \\ 4 Institute for Computational Cosmology, Durham University, South Road, Durham, DH1 3LE, UK \\ 5 CRAL, Université Lyon-1, 9 Av. Charles André, 69561 St Genis Laval, France \\ 6 UK Astronomy Technology Centre, Royal Observatory, Blackford Hill, Edinburgh, EH9 3HJ, UK \\ 7 Harvard-Smithsonian Center for Astrophysics, 60 Garden Street, Cambridge, MA 02138, USA \\ 8 Institute for Astronomy, University of Hawaii, 2680 Woodlawn Dr, Honolulu, HI 96822, USA \\ ${ }^{9}$ Institut d'Astrophysique de Paris, UPMC and CNRS, 98bis Bd. Arago, 75014 Paris, France \\ 10 ASTRON, PO Box 2, 2990 AA Dwingeloo, The Netherlands \\ 11 Geneva Observatory, Université de Genève, 51 chemin des Maillettes, 1290 Versoix, Switzerland \\ 12 LAM, CNRS - Université Aix-Marseille, 38 rue F. Joliot-Curie, 13388 Marseille Cedex 13, France \\ 13 Academia Sinica Institute of Astronomy and Astrophysics (ASIAA), PO Box 23-141, 10617 Taipei, Taiwan \\ 14 Dep. de Astrofisica, Facultad de CC. Fisicas, Universidad Complutense de Madrid, 28040 Madrid, Spain \\ 15 School of Physics and Astronomy, University of Birmingham, Edgbaston, Birmingham B15 2TT, UK \\ 16 Leiden Observatory, Leiden University, PO Box 9513, 2300 RA Leiden, The Netherlands
}

Received 28 December 2011 / Accepted 16 January 2012

\section{ABSTRACT}

\begin{abstract}
During our Herschel Lensing Survey (HLS) of massive galaxy clusters, we have discovered an exceptionally bright source behind the $z=0.22$ cluster Abell 773, which appears to be a strongly lensed submillimeter galaxy (SMG) at $z=5.2429$. This source is unusual compared to most other lensed sources discovered by Herschel so far, because of its higher submm flux ( 200 mJy at $500 \mu \mathrm{m})$ and its high redshift. The dominant lens is a foreground $z=0.63$ galaxy, not the cluster itself. The source has a far-infrared (FIR) luminosity of $L_{\mathrm{FIR}}=1.1 \times 10^{14} / \mu L_{\odot}$, where $\mu$ is the magnification factor, likely $\sim 11$. We report here the redshift identification through CO lines with the IRAM-30 m, and the analysis of the gas excitation, based on $\mathrm{CO}(7-6), \mathrm{CO}(6-5), \mathrm{CO}(5-4)$ detected at IRAM and the $\mathrm{CO}(2-1)$ at the EVLA. All lines decompose into a wide and strong red component, and a narrower and weaker blue component, $540 \mathrm{~km} \mathrm{~s}^{-1}$ apart. Assuming the ultraluminous galaxy (ULIRG) CO-to- $\mathrm{H}_{2}$ conversion ratio, the $\mathrm{H}_{2}$ mass is $5.8 \times 10^{11} / \mu M_{\odot}$, of which one third is in a cool component. From the $\mathrm{CI}\left({ }^{3} \mathrm{P}_{2}-{ }^{3} \mathrm{P}_{1}\right)$ line we derive a $\mathrm{CI} / \mathrm{H}_{2}$ number abundance of $6 \times 10^{-5}$ similar to that in other ULIRGs. The $\mathrm{H}_{2} \mathrm{O}_{p}(2,0,2-1,1,1)$ line is strong only in the red velocity component, with an intensity ratio $I\left(\mathrm{H}_{2} \mathrm{O}\right) / I(\mathrm{CO}) \sim 0.5$, suggesting a strong local FIR radiation field, possibly from an active nucleus (AGN) component. We detect the [NII] $205 \mu \mathrm{m}$ line for the first time at high- $z$. It shows comparable blue and red components, with a strikingly broad blue one, suggesting strong ionized gas flows.
\end{abstract}

Key words. galaxies: evolution - galaxies: high-redshift - galaxies: ISM - infrared: galaxies - submillimeter: galaxies galaxies: individual: HLSJ091828.6+514223

\section{Introduction}

At high redshift, $z \sim 1-3$, dusty luminous infrared galaxies are thought to dominate the history of cosmic star formation (Dole et al.2006; Wardlow et al.2011). At these epochs, star formation was occuring mainly in massive galaxies, a tendency dubbed downsizing in galaxy formation scenarios (e.g. Cowie etal. 1996; Heavens et al.2004; Pérez-Gonzalez et al.2008; Magnelli etal.2009, 2011). These massive starbursts might correspond to the formation phase of the luminous ellipticals seen in high-density regions today (Genzel et al.2003; Swinbank et al.2006). They are therefore essential for understanding the main processes of galaxy formation and evolution. In particular, atomic and molecular lines are crucial clues in these systems for tackling star formation and AGN (Active Galaxy Nucleus) activities (Riechers et al.2010, 2011; Danielson et al. 2011).

Owing to the negative K-correction, the number of submillimeter galaxies (SMGs) does not need to peak at $z \sim 1-3$, indeed, if they exist, they should be as easy to detect at $z=8$ as they are at $z=1-3$. Their observed dearth is probably due to evolution of gas fraction and halo mass (Lacey et al. 2010; Hickox et al. 2012). To be able to make a census of $z \sim 5$ SMG and compare their number with lower- $z$ SMGs, we use Herschel to identify candidate high- $z$ SMGs and strong lensing to provide the signal-to-noise and a unique spatial resolution. This technique 
has now proven quite successful in the study of high- $z$ SMG (e.g. Smail et al. 1997; Pelló et al. 1999; Swinbank et al. 2010; Lestrade et al. 2010; Negrello et al. 2010; Lupu et al. 2011; Cox et al. 2011).

Strongly lensed sources are quite rare on the sky, and are serendipitously discovered in large surveys, such as the South Pole Telescope survey (Vieira et al. 2010), the Herschel HERMES (Oliver et al. 2010), or the huge area Herschel-ATLAS (H-ATLAS) survey (Eales et al. 2010). We have undertaken a more targeted survey with Herschel, focusing on galaxy clusters. Our key project, "Herschel Lensing Survey" (HLS-deep), has the goal to deeply image 44 massive clusters of galaxies with PACS and SPIRE (Egami et al. 2010; Rex et al. 2010), and continues with a SPIRE snapshot survey (HLS-snapshot) of 300 clusters. The unique aim of the HLS survey is to find clusterlensed sources, as opposed to galaxy-lensed objects found in field surveys such as H-ATLAS. These cluster-lensed sources suffer less from differential amplification than galaxy-lensed sources, while the dense environment of clusters increases the probability of galaxy lensing (Smail et al. 2007).

The HLS-deep survey is now fully executed and the source behind Abell 773 is the strongest source at $500 \mu \mathrm{m}$, among the 44 clusters surveyed. Together with the $z=2.78$ lensed galaxy in the Bullet cluster (Rex et al. 2010), it is one of only two HLS-deep SPIRE sources with a peak flux density at $500 \mu \mathrm{m}$ above $100 \mathrm{mJy}$. It is located far from the cluster center $\left(\sim 4.5^{\prime}=1 \mathrm{Mpc}\right)$, and likely lensed by a single galaxy.

We present in this letter the continuum and molecular line observations of the Abell 773 SMG, deriving its gas excitation and physical properties. Section 2 reports the discovery of the SMG from our SPIRE and SMA data, IRAM spectroscopic observations to derive its redshift, with detections of $\mathrm{CO}, \mathrm{C}, \mathrm{H}_{2} \mathrm{O}$, [NII] lines and $\mathrm{CO}(2-1)$ with EVLA. In Sect. 3 we model the data to infer the dust and gas content and its general properties. For distances we adopted the $\Lambda$-CDM concordance model: $H_{0}=71 \mathrm{~km} \mathrm{~s}^{-1} \mathrm{Mpc}^{-1}, \Omega_{\mathrm{M}}=0.27$ and $\Omega_{\Lambda}=0.73$.

\section{Observations}

\subsection{Herschel and SMA}

SPIRE observations were conducted using the "large-map mode", with orthogonal cross-linked scans producing $17^{\prime} \times 17^{\prime}$ coverage in each of the three SPIRE bands. The maps include 20 repetitions with a total observing time of $1.7 \mathrm{~h}$, giving an onsource integration time of $\sim 2000 \mathrm{~s}$. The data were reduced using the Herschel interactive pipeline environment (HIPE v5.0). The maps were produced with the standard naive map maker, but include additional turnaround data with scan speeds $>0.5^{\prime \prime} / \mathrm{s}$ to increase the depth of the outer regions of the maps. For more details see Egami et al. (2010).

HLSJ091828.6+514223 was discovered in the SPIRE map of the $z=0.22$ cluster Abell 773 (Barrena et al. 2007), shown in Fig. 1. Its position is $\alpha_{2000}=09^{\mathrm{h}} 18^{\mathrm{m}} 28.6^{\mathrm{s}}$ and $\delta_{2000}=$ $51^{\circ} 42^{\prime} 23.3^{\prime \prime}$, too far away to be covered by PACS or Spitzer images of the cluster. It lies close to an optical source (Fig. 1), which is likely the lensing galaxy. HLSJ091828.6+514223 has an SED (spectral energy distribution) peaking at $500 \mu \mathrm{m}$, with $F_{500}=203 \mathrm{mJy}$, suggesting a high redshift. The $\mathrm{mm} / \mathrm{submm}$ contribution of the foreground galaxies is negligible.

The Submillimeter Array (SMA) observed the source at $235 \mathrm{GHz}(1.3 \mathrm{~mm})$ on $15 \mathrm{Jun} .2011$ with precipitable water vapor (pwv) between 1.5 and $4 \mathrm{~mm}$ during $62 \mathrm{~min}$ on-source, with $8 \mathrm{GHz}$ total bandwidth. The source was reobserved at

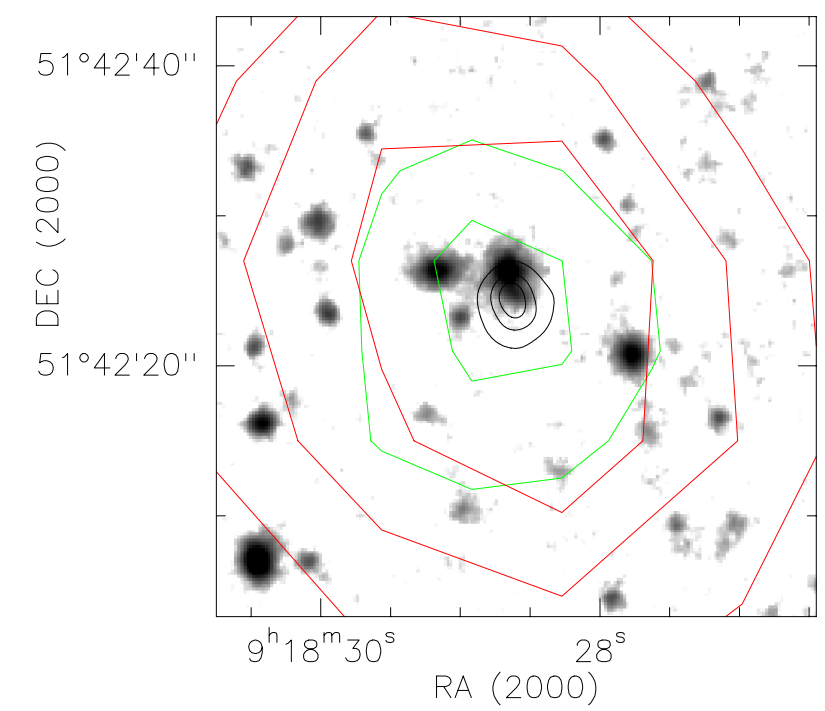

Fig. 1. SPIRE $250 \mu \mathrm{m}$ (green, beam 18"), $500 \mu \mathrm{m}$ (red, 37") and SMA $880 \mu \mathrm{m}$ (black, $2^{\prime \prime}$ ) overplotted on the Subaru (suprime cam) V-image of HLSJ091828.6+514223. The latter shows the lensing galaxy at the center, slightly north of the SMA peak. The SMA contours start at $1 \sigma$ and increase in steps of $4 \sigma$. The EVLA CO(2-1) contours are peaked at the same position and have the same N-S elongation.

Table 1. Flux $S_{v}(\mathrm{mJy})$ for HLSJ091828.6+514223, with IRAM$30 \mathrm{~m}(\mathrm{I})$, SMA (S) and Herschel (H).

\begin{tabular}{lcccccc}
\hline \hline $3 \mathrm{~mm}$ & $2 \mathrm{~mm}$ & $1.3 \mathrm{~mm}$ & $0.88 \mathrm{~mm}$ & $500 \mu \mathrm{m}$ & $350 \mu \mathrm{m}$ & $250 \mu \mathrm{m}$ \\
$\mathrm{I}$ & $\mathrm{I}$ & $\mathrm{S}$ & $\mathrm{S}$ & $\mathrm{H}$ & $\mathrm{H}$ & $\mathrm{H}$ \\
$<2$ & $15 \pm 7$ & $55 \pm 7$ & $125 \pm 8$ & $203 \pm 9$ & $168 \pm 8$ & $85 \pm 8$ \\
\hline
\end{tabular}

Notes. Not including absolute flux calibration uncertainties.

$341 \mathrm{GHz}(880 \mu \mathrm{m})$ on 9 Dec. 2011, during $330 \mathrm{~min}$ on-source and pwv of $1.5 \mathrm{~mm}$. Phase and amplitude gain variations with time were monitored using quasars $0927+390$ and 0920+446, and the flux scale was determined using Titan (Table 1). The source is slightly resolved at $1.3 \mathrm{~mm}$ by the $4.6 \times 2.8^{\prime \prime}$ beam, and resolved at $880 \mu \mathrm{m}$ by the $2.1 \times 2.0^{\prime \prime}$ beam, with an $\mathrm{N} / \mathrm{S}$ extension (Fig. 1).

\subsection{IRAM and EVLA}

To measure the redshift of the source, we used the strategy developed by Weiss et al. (2009) and Lestrade et al. (2010) with the multi-band heterodyne receiver EMIR at the IRAM 30-m telescope. At the time of observations (26-27 Sep. and 6 Oct. 2011), one setup with the $3 \mathrm{~mm}$ receivers provided $7.43 \mathrm{GHz}$ of instantaneous, dual linear polarization bandwidth. We then covered the $35 \mathrm{GHz}$, from 80 to $115 \mathrm{GHz}$, in five setups with some overlaps, integrating about one hour per setup, in wobbler-switching mode. The precipitable water vapor was between 2 and $5 \mathrm{~mm}$, and the system temperatures was $90-100 \mathrm{~K}$ at $3 \mathrm{~mm}$ and $200 \mathrm{~K}$ at $1.3 \mathrm{~mm}$. We used two backends, the $4 \mathrm{MHz}$ filter-banks, and the WILMA autocorrelator, which provides a spectral resolution of $2 \mathrm{MHz}$. Pointing and focus offsets, determined once every two hours, were more accurate than $3^{\prime \prime}$. Continuum levels were obtained at $2 \mathrm{~mm}$ and $1.3 \mathrm{~mm}$ (and an upper limit at $3 \mathrm{~mm}$, cf. Fig. 2 and Table 1). At $1.3 \mathrm{~mm}$, we detected $40 \pm 5 \mathrm{mJy}$, slightly lower than, but still compatible with the SMA value.

The search revealed a line only at the fourth setup, at 92.3 GHz. The fifth setup gave a second line at $110 \mathrm{GHz}$, 
F. Combes et al.: A bright $z=5.2$ lensed submillimeter galaxy in the field of Abell 773

Table 2. Observed line parameters toward HLSJ091828.6+514223.

\begin{tabular}{|c|c|c|c|c|c|c|c|c|}
\hline Line & $\begin{array}{c}v_{\mathrm{obs}} \\
{[\mathrm{GHz}]}\end{array}$ & $\begin{array}{c}T_{\mathrm{mb}} \\
{[\mathrm{mK}]}\end{array}$ & $\begin{array}{c}S_{v} \\
{[\mathrm{mJy}]}\end{array}$ & $\begin{array}{l}\Delta V_{\mathrm{FWHM}} \\
{\left[\mathrm{km} \mathrm{s}^{-1}\right]}\end{array}$ & $\begin{array}{c}I \\
{\left[\mathrm{Jy} \mathrm{km} \mathrm{s}^{-1}\right]}\end{array}$ & $\begin{array}{c}V^{*} \\
{\left[\mathrm{~km} \mathrm{~s}^{-1}\right]}\end{array}$ & $\begin{array}{c}L^{\prime} / 10^{10} \\
{\left[\mathrm{~K} \mathrm{~km} \mathrm{~s}^{-1} \mathrm{pc}^{2}\right]}\end{array}$ & Telescope \\
\hline $\mathrm{CO}(2-1)$ blue & 36.9280 & & $3.1 \pm 0.7$ & $230 \pm 60$ & $0.8 \pm 0.1$ & $-530 \pm 20$ & $17 . \pm 2$ & EVLA \\
\hline red & & & $4.0 \pm 0.7$ & $510 \pm 30$ & $2.2 \pm 0.1$ & $-20 \pm 20$ & $55 . \pm 3$ & \\
\hline $\begin{array}{l}\mathrm{CO}(5-4) \text { blue } \\
\text { red }\end{array}$ & 92.3077 & $\begin{array}{l}1.7 \pm 0.3 \\
2.7 \pm 0.3\end{array}$ & $\begin{array}{c}8.6 \pm 1.5 \\
13.4 \pm 1.5\end{array}$ & $\begin{array}{l}110 \pm 30 \\
540 \pm 40\end{array}$ & $\begin{array}{l}1.0 \pm 0.2 \\
7.7 \pm 0.5\end{array}$ & $\begin{array}{l}-540 \pm 12 \\
-5 \pm 17\end{array}$ & $\begin{array}{l}\text { 4. } \pm 1 \text {. } \\
30 . \pm 2 \text {. }\end{array}$ & IRAM-30 m \\
\hline $\begin{array}{l}\mathrm{CO}(6-5) \text { blue } \\
\text { red }\end{array}$ & 110.7615 & & $\begin{array}{l}11 . \pm 2 \\
16 \pm 2\end{array}$ & & $\begin{array}{l}1.9 \pm 0.4 \\
8.3 \pm 0.6\end{array}$ & $\begin{array}{c}-540 \pm 25 \\
0 \pm 17\end{array}$ & $\begin{array}{l}5 . \pm 1 \text {. } \\
23 . \pm 2 \text {. }\end{array}$ & IRAM-30 m \\
\hline $\begin{array}{l}\mathrm{CO}(7-6) \text { blue } \\
\text { red }\end{array}$ & 129.2111 & $\begin{array}{l}2.0 \pm 0.3 \\
2.9 \pm 0.3\end{array}$ & $\begin{array}{c}9.7 \pm 1.5 \\
14.3 \pm 1.5\end{array}$ & $\begin{array}{l}150 \pm 20 \\
560 \pm 20\end{array}$ & $\begin{array}{l}1.5 \pm 0.2 \\
8.5 \pm 0.3\end{array}$ & $\begin{array}{c}-510 \pm 30 \\
4 \pm 10\end{array}$ & $\begin{array}{l}3 . \pm .5 \\
17 . \pm 1 \text {. }\end{array}$ & $\begin{array}{l}\text { IRAM-30 m } \\
\text { and PdBI }\end{array}$ \\
\hline $\begin{array}{l}\mathrm{C} \mathrm{I}\left({ }^{3} \mathrm{P}_{2}-{ }^{3} \mathrm{P}_{1}\right) \text { blue } \\
\text { red }\end{array}$ & 129.6422 & $\begin{array}{l}1.6 \pm 0.3 \\
1.7 \pm 0.3\end{array}$ & $\begin{array}{l}8.3 \pm 1.5 \\
8.8 \pm 1.5\end{array}$ & $\begin{array}{l}150 \pm 20 \\
530 \pm 40\end{array}$ & $\begin{array}{l}1.4 \pm 0.2 \\
4.9 \pm 0.3\end{array}$ & $\begin{array}{c}-530 \pm 9 \\
20 \pm 14\end{array}$ & $\begin{array}{l}2.8 \pm .5 \\
10 . \pm 1\end{array}$ & $\begin{array}{l}\text { IRAM-30 m } \\
\text { and PdBI }\end{array}$ \\
\hline $\mathrm{H}_{2} \mathrm{O}_{p}(2,0,2-1,1,1)$ & 158.2481 & $2.2 \pm 0.5$ & $11 \pm 2$ & $400 \pm 60$ & $4.8 \pm 0.7$ & $-17 \pm 30$ & $6.5 \pm 0.8$ & IRAM-30 m \\
\hline $\begin{array}{l}\text { NII blue } \\
\text { red }\end{array}$ & 234.0469 & $\begin{array}{l}1.6 \pm 0.2 \\
1.7 \pm 0.2\end{array}$ & $\begin{array}{c}8 \pm 1 \\
8.7 \pm 1\end{array}$ & $\begin{array}{l}410 \pm 90 \\
340 \pm 60\end{array}$ & $\begin{array}{l}3.5 \pm 0.6 \\
3.2 \pm 0.5\end{array}$ & $\begin{array}{l}-620 \pm 35 \\
-33 \pm 30\end{array}$ & $\begin{array}{l}2.2 \pm 0.4 \\
2.0 \pm 0.4\end{array}$ & IRAM-30 m \\
\hline
\end{tabular}

Notes. Data and errors are derived from Gaussian fits for the two V-components. ${ }^{*}$ The velocity is given relative to $z=5.2429$ (centered on the red component of the $\mathrm{CO}(6-5)$ line).

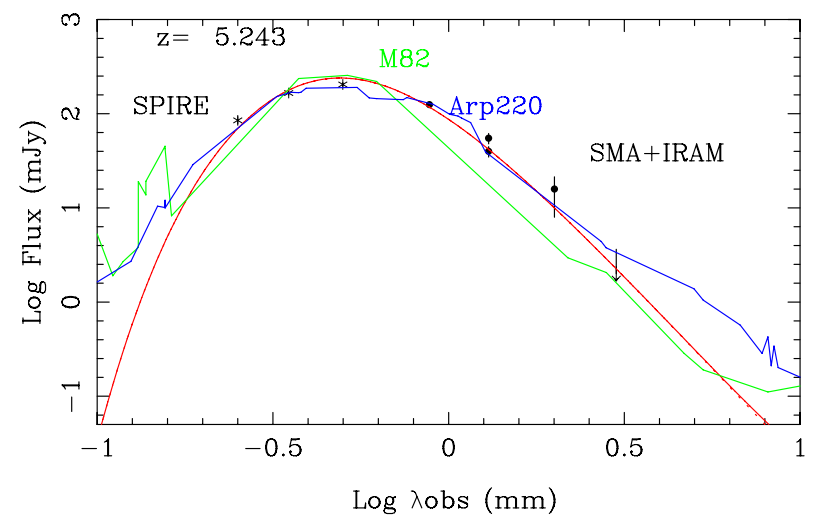

Fig. 2. SPIRE (250, 350 and $500 \mu \mathrm{m})$, SMA $(0.88$ and $1.3 \mathrm{~mm})$ and IRAM $(1.3,2$ and $3 \mathrm{~mm})$ photometric data for HLSJ091828.6+514223, superposed on our model (red line) of a single big grain component at $T_{\mathrm{d}}=52 \mathrm{~K}(\beta=2)$. The M 82 and Arp. 220 SED from NED are also plotted. Our source has an SED very similar to Arp. 220.

suggesting a redshift, which was then confirmed by tuning the $2 \mathrm{~mm}$ receiver at $129 \mathrm{GHz}$, the three detected lines thus being ${ }^{12} \mathrm{CO} J=5-4, J=6-5$ and $J=7-6$ transitions, indicating a redshift of 5.2429. Other lines were searched for, such as dense tracers $\mathrm{HCN}$ and $\mathrm{HCO}^{+}$at $3 \mathrm{~mm}$, water at $2 \mathrm{~mm}$, and the [NII] $205 \mu \mathrm{m}$ line at $1.3 \mathrm{~mm}$.

Around $1 \mathrm{~h}$ of integration was also obtained in $\mathrm{CO}(2-1)$ using the Expanded Very Large Array (Perley et al. 2011), with observations and data reduction following those reported by Ivison et al. (2011).

Figure 3 displays all detected lines, and results of the Gaussian fits are reported in Table 2.

\section{Results}

\subsection{Lens modeling}

The $V$ and $i$ images obtained with the Suprime Cam at Subaru shows the presence of a foreground galaxy at $\sim 1.2^{\prime \prime}$ north of the SMG source. A $3 \times 10$ min spectrum was taken on KeckII with DEIMOS 600 l/mm grating at $7200 \AA$ with the GG455 filter, centered on the foreground galaxy using a $1^{\prime \prime}$ slit at $\mathrm{PA}=14^{\circ}$ in $\sim 1^{\prime \prime}$ seeing. The spectroscopic redshift of the lens was measured as $z=0.63 \pm 0.005$, from CaII-HK lines and the G band.

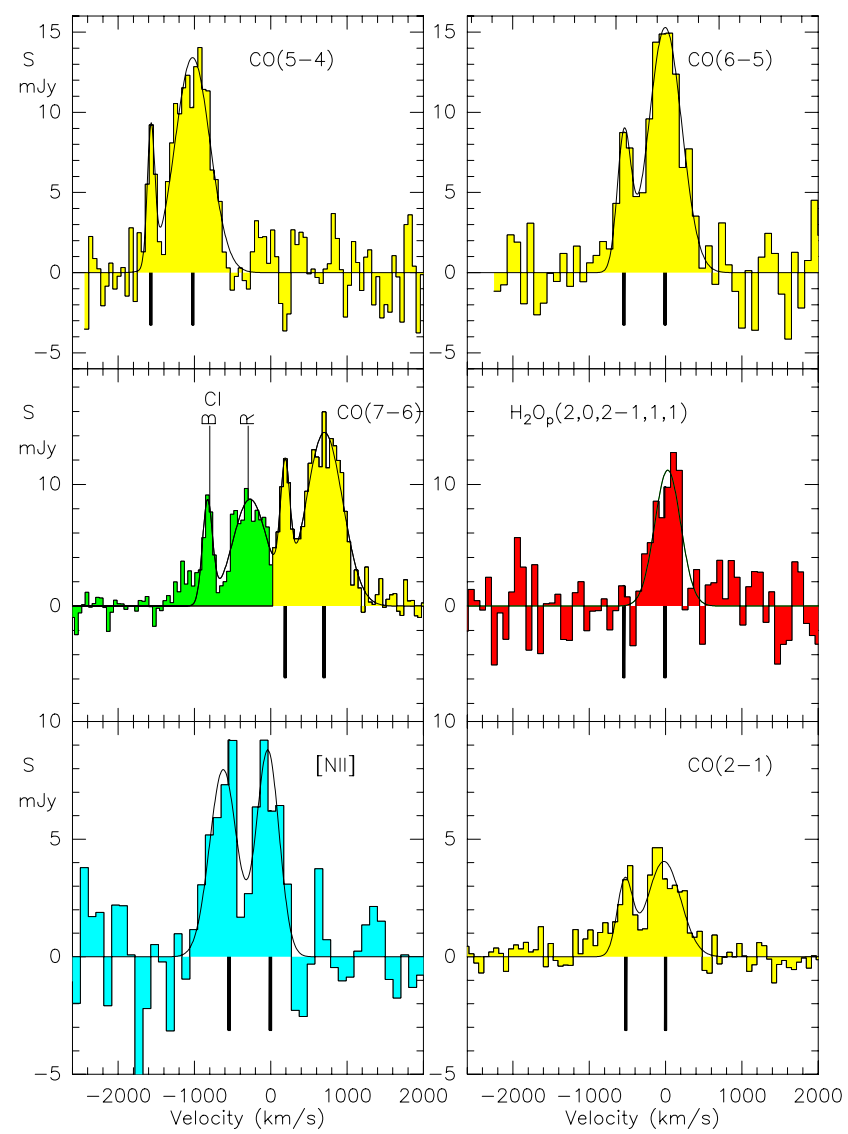

Fig. 3. Four $\mathrm{CO}$ lines, with the $\mathrm{C}\left({ }^{3} \mathrm{P}_{2}-{ }^{3} \mathrm{P}_{1}\right), \mathrm{H}_{2} \mathrm{O}_{p}(2,0,2-1,1,1)$ and $[\mathrm{NII}]$ lines. All spectra are from the IRAM-30 m, except for the $\mathrm{CI} / \mathrm{CO}$ (7-6) from the PdBI, and $\mathrm{CO}(2-1)$ from EVLA. Upper vertical lines indicate the red $(\mathrm{R})$ or blue $(\mathrm{B})$ components for CI, and lower vertical lines indicate the same for all others. All panels have the same velocity scale (translated for $\mathrm{CO}(5-4)$ and $\mathrm{CO} / \mathrm{CI}$ ). The blue component of the [NII] line is much stronger and broader than for the $\mathrm{CO}$ lines, suggesting a strong ionized gas flow. Exact velocities are detailed in Table 2.

Adopting an apparent size for the lensed source of $\sim 2$ " from the SMA maps, we have used LENsTool (Kneib et al. 1993) to produce Einstein rings or crosses of this size, and estimate a magnification $\mu \sim 11$ (sum of the images) for a lens at $z=0.63$. Since 


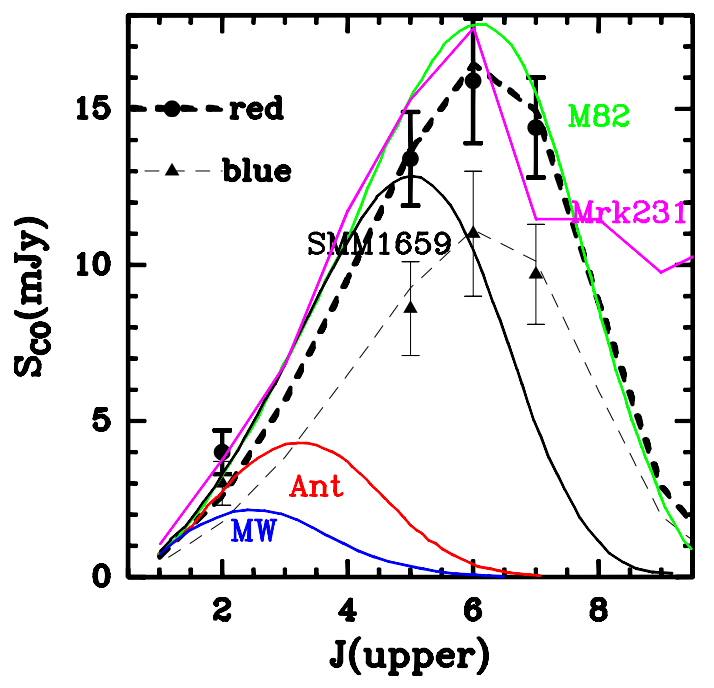

Fig. 4. Peak CO line fluxes for the red (dots) and blue component (triangles), with the best LVG model (bold and light dashed lines, respectively) computed with a density $n_{\mathrm{H}_{2}}=3.5 \times 10^{3} \mathrm{~cm}^{-3}, T_{\mathrm{k}}=45 \mathrm{~K}$, and a column density $N(\mathrm{CO})=10^{18} \mathrm{~cm}^{-2} / \mathrm{km} \mathrm{s}^{-1}$. The light dashed line is related to the bold line in a ratio of 0.8 to 1 . The color lines represent $\mathrm{CO}$ data from other galaxies (e.g. Weiss et al. 2007; Danielson et al. 2011).

the images are not identified yet, we estimate that 11 is the maximum amplification, whose range is 5-11. The cluster does not boost the magnification by more than 10\% (Richard et al. 2010).

\subsection{Dust emission}

Table 1 displays the available photometric data from SPIRE, SMA and the IRAM-30 m. We fitted the data in Fig. 2 with a single optically thin big grain component at an average temperature $T_{\mathrm{d}}=52 \mathrm{~K}$, an emissivity slope $\beta=2$, and a mass absorption coefficient $\kappa=50 \mathrm{~cm}^{2} / \mathrm{g}$ at $100 \mu \mathrm{m}$. We derived the total FIR $(8-1000 \mu \mathrm{m})$ luminosity $L_{\mathrm{FIR}}=1.1 \times 10^{14} / \mu L_{\odot}$, and the star-formation rate $S F R=1.8 \times 10^{4} / \mu M_{\odot} / y r$, applying the relation from Kennicutt (1998). Fitting with other dust models, such as Chary \& Elbaz (2001), leads to the same luminosity, within the uncertainties. The mass of the dust is then $M_{\text {dust }}=5.0 \times 10^{9} / \mu M_{\odot}$, which for a gas-to-dust mass ratio of 150 leads to $M_{\text {gas }}=7.6 \times 10^{11} / \mu M_{\odot}$. Other fits are possible, although less good, with lower values of $\beta$ (down to 1.5), higher dust temperatures and lower dust masses, down by a factor 4 .

\subsection{CO lines}

Four CO lines are clearly detected in two components, as shown in Fig. 3. The peak flux of the stronger red component is modeled in Fig. 4 with an LVG (large velocity gradient) code, varying the $\mathrm{H}_{2}$ volume density, the $\mathrm{CO}$ column density and the kinetic temperature (Combes et al. 1999). The blue component has a peak intensity lower by $25 \%$, whatever the $J$ level, within the error bars. The three high- $J$ CO lines are well fitted by a single excitation component, peaking at the $J=6$ level. We found the best fit with a density $n_{\mathrm{H}_{2}}=3.5 \times 10^{3} \mathrm{~cm}^{-3}, T_{\mathrm{k}}=45 \mathrm{~K}$, and a column density $N(\mathrm{CO})=10^{18} \mathrm{~cm}^{-2} / \mathrm{km} \mathrm{s}^{-1}$. The solution is somewhat degenerate, between low $\mathrm{H}_{2}$ density and higher $\mathrm{CO}$ column density, and we favor optically thick $\mathrm{CO}$ emission. PDR (photodissociation region) models fit as well, the $\mathrm{CO}$ being emitted by small and dense optically thick clouds. Observations of higher $J$ CO lines are required to distinguish between PDR and XDR (Xray-dissociated region), where CO is emitted by a more extended and diffuse hot medium (Meijerink et al. 2007). The observed $\mathrm{CO}(2-1)$ flux is $50 \%$ higher than predicted by the high-excitation component, suggesting the presence of a cooler component, containing one third of the mass. Adopting the conversion factor $M\left(\mathrm{H}_{2}\right) / L_{\mathrm{CO}(1-0)}^{\prime}=0.8 M_{\odot} /\left(\mathrm{K} \mathrm{km} \mathrm{s}^{-1} \mathrm{pc}^{2}\right)$ of ULIRGs (ultraluminous galaxies, e.g. Solomon et al. 1997), we derive from the $\mathrm{CO}(2-1)$ integrated flux an $\mathrm{H}_{2}$ mass of $5.8 \times 10^{11} / \mu M_{\odot}$. This mass is 0.8 times that derived from the dust emission, but there are still uncertainties in the conversion ratio to apply. The derived ratio $L_{\mathrm{FIR}} / L_{\mathrm{CO}(3-2)}^{\prime} \sim 260$, compatible with other (U)LIRGs (Iono et al. 2009).

\subsection{Atomic carbon}

Since the $\mathrm{C}_{\mathrm{I}}\left({ }^{3} \mathrm{P}_{1}-{ }^{3} \mathrm{P}_{0}\right)$ line is not available in the receiver range, we have observed the $\mathrm{CI}_{\mathrm{I}}\left({ }^{3} \mathrm{P}_{2}-{ }^{3} \mathrm{P}_{1}\right)$ with the $30 \mathrm{~m}$, and confirmed it later with the Plateau de Bure (PdBI), with a better signal-tonoise ratio (Boone et al. , in prep.). The latter is shown in Fig. 3, with the two velocity components detailed in Table 2 . From an adopted excitation temperature of $T_{\mathrm{ex}}=40 \mathrm{~K}$, and a total luminosity $L_{\mathrm{CI}\left({ }^{3} \mathrm{P}_{2}{ }^{-}{ }^{3} \mathrm{P}_{1}\right)}^{\prime}=12.8 \times 10^{10} \mathrm{~K} \mathrm{~km} \mathrm{~s}^{-1} \mathrm{pc}^{2}$, we derive a carbon mass of $M_{\mathrm{CI}}=2 \times 10^{8} / \mu M_{\odot}$ (Weiss et al. 2005). This yields a $\mathrm{C} \mathrm{I} / \mathrm{H}_{2}$ number abundance of $6 \pm 2 \times 10^{-5}$, comparable to other high-z SMGs (e.g. Danielson et al. 2011).

\subsection{Water}

ISO and Herschel observations have revealed that far-infrared $\mathrm{H}_{2} \mathrm{O}$ lines are surprisingly strong with respect to CO in ULIRGs, in particular those containing an AGN (Arp 220, Cloverleaf, Mrk 231, Gonzalez-Alfonso et al. 2004, 2008; Bradford et al. 2009; van der Werf etal.2010). Water is thought to evaporate from grains in shocked regions, in dense hot cores due to cosmic rays or possibly X-rays near AGN (Gonzalez-Alfonso et al. 2010). In Mrk231, van der Werf et al. (2010) have shown that starbursts and PDR alone cannot explain the high- $J$ excitation of the $\mathrm{CO}$ lines, nor the $\mathrm{H}_{2} \mathrm{O} / \mathrm{CO}$ ratios, and a very strong FIR radiation field or XDR conditions related to AGN are required. The associations of starbursts with AGN might be even more frequent at high redshift, and a strong $\mathrm{H}_{2} \mathrm{O}$ line has been detected at $z=2.3$ (Omont et al. 2011) and $z=3.91$ (e.g. van der Werf et al. 2011).

The water line detected in HLSJ091828.6+514223 is the highest flux line expected from the mixed model of Gonzalez-Alfonso \& Cernicharo (1999), including collisional excitation and far-infrared pumping. We did not detect the $\mathrm{H}_{2} \mathrm{O}_{o}(1,1,0-1,0,1)$, with an upper limit 3 times lower than the $\mathrm{H}_{2} \mathrm{O}_{p}(2,0,2-1,1,1)$ line, which is compatible with the model (expected ratio of 4 ). The water lines are very optically thick, but information comes from their ratio to the $\mathrm{CO}$ lines, which here is $I\left(\mathrm{H}_{2} \mathrm{O}\right) / I(\mathrm{CO}) \sim 0.5$, equal to the ratio observed in Mrk231 or the Cloverleaf quasar at $z=2.6$, SDP.17b at $z=2.3$, and a little lower than in APM08279 at $z=3.91$ (van der Werf et al. 2011, and references therein). In all these galaxies, this ratio is 1-2 orders of magnitude higher than in Orion bar. This suggests the existence of a hot dense component, or an XDR in HLSJ091828.6+514223.

\subsection{The $[N I I] 205 \mu m$ line}

Figure 3 displays the spectrum of the ${ }^{3} \mathrm{P}_{1}->{ }^{3} \mathrm{P}_{0}$ ground state fine structure line of ionized nitrogen, smoothed to a resolution of $100 \mathrm{~km} \mathrm{~s}^{-1}$. The two velocity components are clearly 
Table 3. Line upper limits at $3 \sigma$ in channels of $140 \mathrm{~km} \mathrm{~s}^{-1}$.

\begin{tabular}{lcc}
\hline \hline Line & $v_{\text {obs }}(\mathrm{GHz})$ & $S_{v}(\mathrm{mJy})^{*}$ \\
\hline $\mathrm{HCN} \& \mathrm{HCO}^{+}(6-5)$ & $\sim 86$. & $<4$. \\
${ }^{13} \mathrm{CO}(5-4)$ & 88.2484 & $<10$. \\
$\mathrm{H}_{2} \mathrm{O}_{o}(1,1,0-1,0,1)$ & 89.2111 & $<4$. \\
$\mathrm{CS}(11-10)$ & 86.2882 & $<10$. \\
\hline
\end{tabular}

detected, and the blue component is much broader than for the $\mathrm{CO}$ and $\mathrm{CI}$ lines. This fine structure line, with a low excitation potential, mainly traces HII regions photoionized by OB stars. In the Milky Way, the line appears moderate, with an intensity ratio $[\mathrm{CII}] /[\mathrm{NII}] \sim 10$. The latter ratio is 20 in M 82 (Petuchowski et al. 1994), and 15 in Mrk 231 (Fischer et al.2010). Only upper limits have been reported in high- $z$ galaxies, the most constraining being in the $\mathrm{J} 1148+5251$ quasar at $z=6.4$ (Walter et al. 2009), with $L_{[\mathrm{NII}]} / L_{\mathrm{FIR}}<2 \times 10^{-5}$, and $L_{[\mathrm{NII}]} / L_{\mathrm{CO}(6-5)}<$ 0.9 . Here we detect the line with $L_{[\mathrm{NII}]} / L_{\mathrm{FIR}}=4 \times 10^{-5}$ and $L_{[\mathrm{NII}]} / L_{\mathrm{CO}(6-5)}=0.15$. These values are intermediate between the AGN-dominated Mrk 231, where $L_{[\mathrm{NII}]} / L_{\mathrm{FIR}}=0.8 \times 10^{-5}$, and the starburst $\mathrm{M} 82$, where $L_{[\mathrm{NII}]} / L_{\mathrm{FIR}}=7.5 \times 10^{-5}$. The broad line seen in the [NII] blue velocity component could be related to galactic winds, often seen in AGN/starburst composite ULIRGs, and with higher velocities than in neutral flows (Veilleux et al. 2005; Rupke et al. 2005; Fischer et al. 2010).

\subsection{Other lines}

We have searched for the high-density tracers $\mathrm{HCN}$ and $\mathrm{HCO}^{+}$, in their lowest available level, i.e. $J=6-5$ (Table 3 ). The upper limits found $\mathrm{HCN}(6-5) / \mathrm{CO}(6-5)<0.25$ are not yet constraining, since ULIRGs have on average $\mathrm{HCN} / \mathrm{CO} \sim 0.1$, except for 1 or 2 luminous quasars (Gao \& Solomon 2004).

\section{Discussion and conclusion}

We have estimated an amplification $\mu \lesssim 11$ from LENSTOoL (likely range 5-11). The amplification factor can also be estimated from the trend of the $\mathrm{CO}(1-0)$ FWHM line width with luminosity $L$ (Harris et al. 2012; Bothwell et al., in prep.): $\mu=$ $3.5 *\left(L^{\prime} / 10^{11} \mathrm{~K} \mathrm{~km} \mathrm{~s}^{-1} \mathrm{pc}^{2}\right) *\left(\mathrm{FWHM} / 400 \mathrm{~km} \mathrm{~s}^{-1}\right)^{-1.7}$. This yields $\mu=16 \pm 5$, which is consistent with our earlier estimate, and suggests that the amplification is likely $\sim 11$. Taking into account this magnification, the intrinsic FIR luminosity of the source is still in the $\sim 10^{13} L_{\odot}$ range, corresponding to a hyperluminous source. The detected lines (except water) are divided into two velocity components, which suggests a galaxy merger, and would explain the high derived SFR (Engel et al. 2010).

At these high luminosities, an AGN component is probable, which is supported by the high water emission. However, the flux in the CO ladder peaks at $J=6$ as in starbursts like NGC 253 and M 82, and we have no evidence yet that it is extended to higher $J$ as in powerful AGNs like Mrk 231 or APM08279. The [NII] $205 \mu \mathrm{m}$ line is detected for the first time at high- $z$, and its relative luminosity with respect to $L_{\mathrm{FIR}}$ is intermediate between a starburst (M 82) and an AGN (Mrk 231). Its blue velocity component is much broader than for the $\mathrm{CO}$ lines, which could come from violent flows of ionized gas. The two velocity components have quite different properties (in $\mathrm{H}_{2} \mathrm{O}$, [NII]), favoring an interpretation in terms of two galaxies, with a high velocity difference of $540 \mathrm{~km} \mathrm{~s}^{-1}$. Observations of the higher-J CO lines are needed to complete the gas excitation, and interferometer maps of key lines, such as the [CII] line, will bring more information about the nature of this interesting system.

Acknowledgements. IRAM is supported by INSU/CNRS (France), MPG (Germany) and IGN (Spain). Herschel is an ESA space observatory with instruments provided by European-led PI consortia and with important participation from NASA. Support for this work was provided in part by NASA through an award issued by JPL/Caltech. The SMA is a joint project between the Smithsonian Astrophysical Observatory and the Academia Sinica Institute of Astronomy and Astrophysics and is funded by the Smithsonian Institution and the Academia Sinica. NRAO is operated by Associated Universities Inc., under a cooperative agreement with the National Science Foundation. The Keck observatory, made possible thanks to the generous support of W. M. Keck Foundation, is operated by Caltech, the University of California, and NASA.

\section{References}

Barrena, R., Boschin, W., Girardi, M., \& Spolaor, M. 2007, A\&A, 467, 37 Bradford, C. M., Aguirre, J. E., Aikin, R., et al. 2009, ApJ, 705, 112

Chary, R., \& Elbaz, D. 2001, ApJ, 556, 562

Combes, F., Maoli, R., \& Omont, A. 1999, A\&A, 345, 369

Cowie, L. L., Songaila, A., Hu, E. M., \& Cohen, J. G. 1996, AJ, 112, 839

Cox, P., Krips, M., Neri, R., et al. 2011, ApJ, 740, 63

Danielson, A. L. R., Swinbank, A. M., \& Smail, I. 2011, MNRAS, 410, 1687

Dole, H., Lagache, G., Puget, J.-L., et al. 2006, A\&A, 451, 417

Eales, S., Dunne, L., Clements, D., et al. 2010, PASP, 122, 499

Egami, E., Rex, M., Rawle, T. D., et al. 2010, A\&A, 518, L12

Engel, H., Tacconi, L., Davies, R. L., et al. 2010, ApJ, 724, 233

Fischer, J., Sturm, E., Gonzalez-Alfonso, E., et al. 2010, A\&A, 518, L41

Gao, Y., \& Solomon, P. M. 2004, ApJS, 152, 63

Genzel, R., Baker, A. J., Tacconi, L. J., et al. 2003, ApJ, 584, 633

Gonzalez-Alfonso, E., \& Cernicharo, J. 1999, ApJ, 525, 845

Gonzalez-Alfonso, E., Smith, H., Fischer, J., \& Cernicharo, J. 2004, ApJ, 613, 247

Gonzalez-Alfonso, E., Smith, H., Ashby, M., et al. 2008, ApJ, 675, 303

Gonzalez-Alfonso, E., Fischer, J., Isaak, K., et al. 2010, A\&A, 518, L43

Harris, A. I., et al. 2012, ApJ, submitted

Heavens, A., Panter, B., Jimenez, R., \& Dunlop, J. 2004, Nature, 428, 625

Hickox, R. C., Wardlow, J. L., Smail, I., et al. 2012, MNRAS, in press [arXiv: 1112.0321]

Iono, D., Wilson, C. D., Yun, M. S., et al. 2009, ApJ, 695, 1537

Ivison, R. J., Papadopoulos, P. P., Smail, I., et al. 2011, MNRAS, 412, 1913

Kennicutt, R. 1998, ARA\&A, 38, 189

Kneib, J.-P., Mellier, Y., Fort, B., \& Mathez, G. 1993, A\&A, 273, 367

Lacey, C. G., Baugh, C. M., \& Frenk, C. S., et al. 2010, MNRAS, 405, 2

Lestrade, J.-F., Combes, F., Salomé, P., et al. 2010, A\&A, 522, L4

Lupu, R. E., Scott, K. S., Aguirre, J. E., et al. 2011, ApJ, submitted [arXiv: 1009. 5983]

Magnelli, B., Elbaz, D., Chary, R. R., et al. 2009, A\&A, 496, 57

Magnelli, B., Elbaz, D., Chary, R. R., et al. 2011, A\&A, 528, A35

Negrello, M., Hopwood, R., De Zotti, G., et al. 2010, Science, 330, 800

Oliver, S. J., Wang, L., Smith, A. J., et al. 2010, A\&A, 518, L21

Omont, A., Neri, R., Cox, P., et al. 2011, A\&A, 530, L3

Meijerink, R., Spaans, M., \& Israel, F. P. 2007, A\&A, 793, 811

Pelló, R., Kneib, J. P., Le Borgne, J. F., et al. 1999, A\&A, 346, 359

Pérez-Gonzalez, P. G., Rieke, G. J., Villar, V., et al. 2008, ApJ, 675, 234

Perley, R . A., Chandler, C. J., Butler, B. J., \& Wrobel, J. M. 2011, ApJ, 739, L1

Petuchowski, S. J., Bennett, C. L., Haas, M. R., et al. 1994, ApJ, 427, L17

Rex, M., Rawle, T. D., Egami, E., et al. 2010, A\&A, 518, L13

Richard, J., Smith, G. P., Kneib, J.-P., et al. 2010, MNRAS, 404, 325

Riechers, D., Weiss, A., Walter, F., \& Wagg, J. 2010, ApJ, 725, 1032

Riechers, D., Carilli, C., Walter, F., et al. 2011, ApJ, 733, L11

Rupke, D. S., Veilleux, S., \& Sanders, D. B. 2005, ApJ, 632, 751

Smail, I., Ivison, R. J., \& Blain, A. W. 1997, ApJ, 490, L5

Smail, I., Swinbank, A. M., Richard, J., et al. 2007, ApJ, 654, L33

Solomon, P. M., Downes, D., Radford, S. J. E., \& Barrett, J. W. 1997, ApJ, 478, 144

Swinbank, A. M., Chapman, S. C., Smail, I., et al. 2006, MNRAS, 371, 465

Swinbank, A. M., Smail, I., \& Longmore, S., et al. 2010, Nature, 464, 733 van der Werf, P., Isaak, K., Meijerink, R., et al. 2010, A\&A, 518, L42 van der Werf, P., Berciano Alba, A., Spaans, M., et al. 2011, ApJ, 741, L38

Veilleux, S., Cecil, G., \& Bland-Hawthorn, J. 2005, ARA\&A, 43, 769

Vieira, J. D., Crawford, T. M., Switzer, E. R., et al. 2010, ApJ, 719, 763

Walter, F., Weiss, A., Riechers, D., et al. 2009, ApJ, 691, L1

Wardlow, J. L., Smail, I., Coppin, K. E. K., et al. 2011, MNRAS, 415, 1479

Weiss, A., Downes, D., Henkel, C., \& Walter, F. 2005, A\&A, 429, L25

Weiss, A., Downes, D., Neri, R., et al. 2007, A\&A, 467, 955

Weiss, A., Ivison, R. J., Downes, D., et al. 2009, ApJ, 705, L45 